\title{
ESTABILIDAD DE TENSIÓN EN EL CORTO PLAZO: FENÓMENO, ANÁLISIS Y ACCIONES DE CONTROL
}

\author{
Rosa de Castro Fernández ${ }^{1} \quad$ Horacio Díaz Rojas ${ }^{2}$ \\ Recibido el 26 de noviembre 2001, aceptado el 14 de agosto de 2002
}

\begin{abstract}
RESUMEN
En este trabajo se describe, analiza y simula el problema de estabilidad de tensión en el corto plazo. El análisis se realiza de manera didáctica, razón por la cual se considera un pequeño sistema de prueba y se presentan en detalle los procedimientos metodológicos, así como los modelos dinámicos completos de componentes y equipos. Utilizando Simulink se estudia la dinámica del sistema en el corto plazo, considerando como alternativas de intervención para evitar el colapso de tensión, el desprendimiento de carga y el control de potencia reactiva a través del empleo de bancos de condensadores, controladores estáticos de potencia reactiva o compensadores síncronos.

Los resultados del análisis dinámico muestran que los tiempos asociados a los retardos de la acción de control resultan importantes. En particular, al comparar las alternativas propuestas se observa que el empleo de un compensador síncrono es la mejor de las opciones analizadas, sin embargo también una de las de mayor costo; los bancos de condensadores son una alternativa mucho más económica pero su efectividad es altamente dependiente del tiempo de conexión. En cuanto al desprendimiento de carga, éste resulta mucho más efectivo mientras más rápido se realice y se obtienen mejores resultados si se aplica a cargas asociadas a motores de inducción.
\end{abstract}

Palabras claves: Estabilidad de tensión, compensación de potencia reactiva, simulación dinámica

\begin{abstract}
This paper describes, analyses and simulates short-term voltage stability phenomenon. A didactic approach is used for the analysis, utilizing a small test system, providing a detailed description of the methodological procedures, as well as complete dynamic models of components and equipment used. The study of the short term dynamics of the system is done using Simulink. This implies considering the effect of different reactive control alternatives, such as the use of a capacitor bank, static voltage controller and a synchronous compensator. Besides, load shedding is applied to avoid voltage collapse. The dynamic analysis demonstrates that switching time of reactive power compensation is critical. In particular, the synchronous compensator dynamics is the best of the alternatives analysed, but this also represents a higher cost. On the contrary, the capacitor bank is a less expensive choice, but its effectiveness highly depends on the switching time. As for the load shedding, this is more effective, the sooner it is done. Better results are achieved when load shedding is applied to inductive engines.
\end{abstract}

Keywords: Voltage stability, reactive power compensation, dynamic simulation

\section{INTRODUCCIÓN}

La creciente liberalización de los mercados eléctricos [4] ha llevado a las redes eléctricas a operar cada vez más cerca de sus límites de capacidad de carga, con lo cual surgen nuevas problemáticas, entre ellas el problema de estabilidad de tensión se ha constituido en los últimos años en un tema de fuerte preocupación [1], [3], [11] debido a la importancia de este fenómeno en la seguridad y calidad del suministro.

Básicamente, el problema consiste en mantener la tensión de la red en niveles que garanticen su operación estable, para ello se consideran como alternativas para evitar el colapso de tensión el control de potencia reactiva [5] y /o el desprendimiento de carga [6].

\footnotetext{
1 Universidad Politécnica de Madrid, Departamento de Ingeniería Eléctrica, ETSII, Madrid, España, rcastro@inel.etsii.upm.es

2 Universidad de Tarapacá, Departamento de Electrónica, Casilla 6-D, Arica, Chile, hdiaz@uta.cl
} 
Existen diversas metodologías para enfrentar el estudio del problema de estabilidad de tensión de una red eléctrica, las cuales desde el punto de vista de los intervalos de tiempo considerados pueden clasificarse en estudios de corto plazo o largo plazo. En el primer caso, se analiza la respuesta del sistema durante los primeros segundos después de una gran perturbación, siendo importante considerar la dinámica de motores, controladores estáticos de potencia reactiva (SVC), compensadores síncronos (CS), reguladores automáticos de tensión (AVR), etc., por lo tanto se requiere un modelo dinámico de los componentes, así como un programa de análisis de transitorios. En el segundo caso, se trata de estudiar el comportamiento de la red durante varios minutos, a fin de evaluar, por ejemplo, la respuesta del sistema ante un crecimiento sostenido de la demanda, por lo tanto resulta importante modelar adecuadamente la dinámica de las cargas y la dinámica de los elementos de la red con respuesta más lenta como los transformadores con cambio de tomas (LTC), el control de la sobrexcitación de los generadores, la compensación shunt (C), etc.

El objetivo fundamental de este trabajo es facilitar la comprensión del fenómeno de estabilidad de tensión a todos aquellos que se inicien en el tema, para ello se ha considerado una estructura didáctica a fin de presentar, de manera relativamente sencilla, cómo debe modelarse y estudiarse el problema de estabilidad de tensión. Por esta razón se considera un pequeño sistema de prueba obtenido de [1] y se presentan en detalle los procedimientos metodológicos, así como los modelos completos de componentes y equipos.

En primer lugar, el sistema seleccionado se emplea con la finalidad de presentar los fundamentos básicos del problema de estabilidad de tensión, para ello, se realiza un estudio desde un punto de vista cuasi-estático a fin de detectar e identificar de manera sencilla las principales variables de interés que intervienen en el estudio de colapso de tensión.

Posteriormente, se presenta un análisis de estabilidad de tensión en el corto plazo, modelándose el sistema en estudio considerando la dinámica de los diferentes componentes de la red y se formula una metodología de análisis empleando Simulink. A partir de este modelo se evalúa el efecto que tienen los tiempos de actuación de los mecanismos de control y los tiempos de duración de las contingencias, estudiándose la dinámica del sistema considerando como alternativas de intervención para evitar el colapso de tensión el desprendimiento de carga y el control de potencia reactiva a través del empleo de bancos de condensadores, controladores estáticos de potencia reactiva y compensadores síncronos.

A fin de comprender de mejor forma los modelos y metodologías empleadas, los autores han desarrollado un tutorial que muestra el detalle teórico y los programas utilizados para obtener los resultados publicados. Este tutorial puede descargarse libremente desde Internet, lo que unido al hecho de que Simulink es una herramienta de simulación ampliamente difundida hace posible que se puedan verificar los resultados obtenidos y estudiar otras situaciones particulares no presentadas en este trabajo.

De esta forma el estudio del problema que se presenta facilita la comprensión de los fenómenos dinámicos asociados a la estabilidad de tensión en el corto plazo, los resultados obtenidos demuestran la importancia de éste tipo de análisis para el diseño y selección de controladores o sistemas de protecciones, donde los tiempos de duración de las contingencias y la dinámica de los dispositivos de control de potencia reactiva son factores determinantes que influyen en el problema de estabilidad de tensión de una red eléctrica.

\section{PRESENTACIÓN DEL PROBLEMA}

En la práctica, el problema de estabilidad de tensión ocurre en redes de gran tamaño; siendo necesario muchas veces el desarrollo de un estudio previo que permita evaluar los sectores de la red más sensibles a posibles problemas de colapso de tensión con el objetivo de reducir la red a una de menor tamaño a fin de realizar un estudio práctico del sistema. Las metodologías necesarias para esta evaluación escapan a los objetivos de esta publicación, razón por la cual se utilizará como sistema de estudio la red mostrada en la Fig. 1, la cual corresponde a una red de transmisión simplificada [1] que alimenta una carga de $600 \mathrm{MW}$ desde un gran sistema a través de dos líneas iguales y en paralelo de $230 \mathrm{kV}$. La tensión del nudo de carga (LOAD) se mantiene constante mediante un transformador con cambio automático de tomas (LTC). Se considerará que el sistema está fuertemente cargado y con gran compensación de potencia reactiva en la barra REC. La mitad de la potencia activa es demandada por un motor de inducción y la otra mitad por una carga residencial. El consumo de potencia reactiva del motor se compensa al $80 \%$ por un banco de condensadores.

El problema que se trata de estudiar es el comportamiento de la tensión en la barra REC después de la ocurrencia de un cortocircuito trifásico en el punto medio de una de las líneas, para evaluar la 
posible existencia del fenómeno de colapso de tensión.

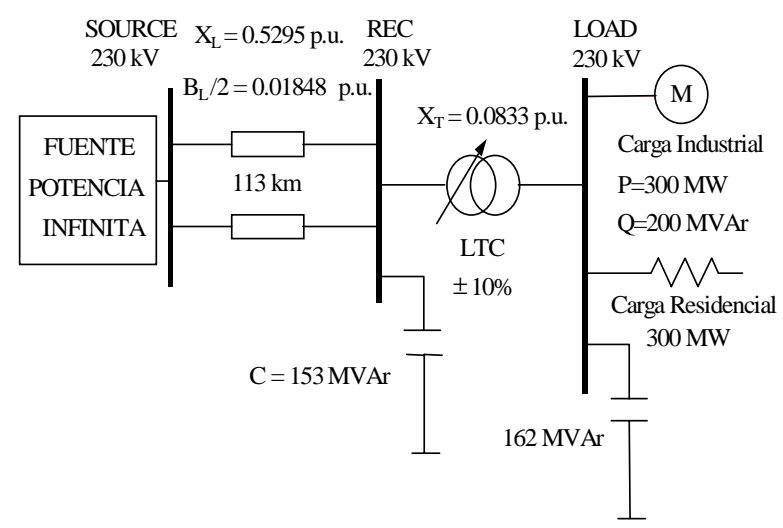

Fig. 1.- Sistema de estudio

También se requiere estudiar el efecto de la duración de las contingencias y el tiempo de actuación de las acciones de control: desprendimiento de carga y control de potencia reactiva en la barra REC considerando bancos de condensadores (C), controladores estáticos de potencia reactiva $(\mathrm{SVC}) \mathrm{o}$ compensadores síncronos (CS)

\section{ESTABILIDAD DE TENSIÓN DESDE UN PUNTO DE VISTA CUASI-ESTÁTICO}

Aun cuando el problema de estabilidad de tensión es de naturaleza dinámica, éste puede simplificarse y modelarse desde un punto de vista de régimen permanente (análisis cuasi-estático) a través del análisis de las curvas P-V o Q-V [8] en una barra de la red. Desde un punto de vista teórico la ventaja de este análisis es que permite identificar de manera sencilla la posible existencia de un problema de estabilidad de tensión.

Para aplicar esta metodología al estudio de la red de la Fig. 1 se analizarán las curvas Q-V en el nudo REC, para ello se necesita trazar en un mismo gráfico la curva Q-V del banco de condensadores y la curva Q-V del sistema. Para modelar de manera estática el comportamiento del sistema en el intervalo de tiempo que existe entre la situación de pre-falta y post-falta, los elementos de la red se modelan de acuerdo a la naturaleza de su comportamiento durante cada uno de los diferentes intervalos de tiempo considerados.

Finalmente, si existe intersección entre estas curvas entonces el sistema se podría considerar como estable, si no fuera así, se dice que existe un problema de colapso de tensión, pues la potencia reactiva inyectada en la red es insuficiente para mantener un nivel de tensión estable.

Para el sistema en estudio se han considerado cinco casos de interés a fin de modelar de manera estática la respuesta del sistema durante el periodo de pre-falta y post-falta. Como se observa en la Fig. 2, el caso 1 corresponde al periodo de tiempo en que el sistema opera de manera estable antes de la ocurrencia de la perturbación (pre-falta) y los casos 2 a 5 corresponden a los diferentes periodos considerados después de la ocurrencia de la perturbación (post-falta); en cada uno de ellos se trata de modelar el comportamiento de los diferentes elementos que constituyen la red a medida que transcurre el tiempo. Por ejemplo, en los casos 2 y 3 se considera que el LTC aún no ha tenido tiempo suficiente para actuar por lo cual su toma se mantiene fija, en ambos casos, la carga residencial se ha modelado a impedancia constante pero la carga industrial en el caso 2 se modela a impedancia constante y en el caso 3 a potencia constante.

A partir del análisis de las curvas Q-V obtenidas para cada uno de los cinco casos considerados, Fig. 3, se puede observar que los casos 1 y 2 serían estables, mientras que en los casos 3 y 4 se observa que el sistema está cercano a una situación crítica, puesto que los niveles de tensión son muy reducidos e incluso puede no existir intersección entre las curvas (colapso). En cualquiera de los casos deben tomarse acciones de control para asegurar que el sistema opere a niveles de tensión aceptables.

Claramente la peor situación (colapso de tensión) corresponde al caso 5 en el cual se ha considerado que después de despejada la falta ha transcurrido el tiempo suficiente para que las cargas vuelvan a comportarse como antes de la perturbación (potencia constante). Es decir, el sistema es incapaz de mantener la demanda de pre-falta con una sola línea aun cuando el LTC actúe.

A partir de los resultados obtenidos en la Fig. 3 se puede observar la importancia de los modelos considerados para los componentes de la red, al igual que la influencia de la potencia reactiva, pues si se aumenta el tamaño el banco de condensadores del nudo REC el problema tendería a resolverse. Sin embargo, se observa que existe una incertidumbre con respecto al tiempo disponible para tomar esta acción de control. Por lo tanto la metodología en base al análisis de las cuervas Q-V es sencilla y permite estimar posibles problemas de estabilidad de tensión, pero no resulta apropiado cuando se requiere hacer un análisis más preciso del problema. Por ejemplo con esta metodología no es posible resolver la segunda parte del problema planteado en cuanto al estudio del efecto del 
tiempo de retardo asociado a las acciones de control Existen diferentes referencias bibliográficas que tratan el tema de la estabilidad de tensión desde un punto de vista dinámico, tal como el que se presenta en [7], donde se realiza un estudio para mostrar el efecto del tiempo de conexión de bancos de condensadores y del desprendimiento de carga en un sistema equivalente chileno, demostrándose la importancia de la variable tiempo en la toma de la acción de control.

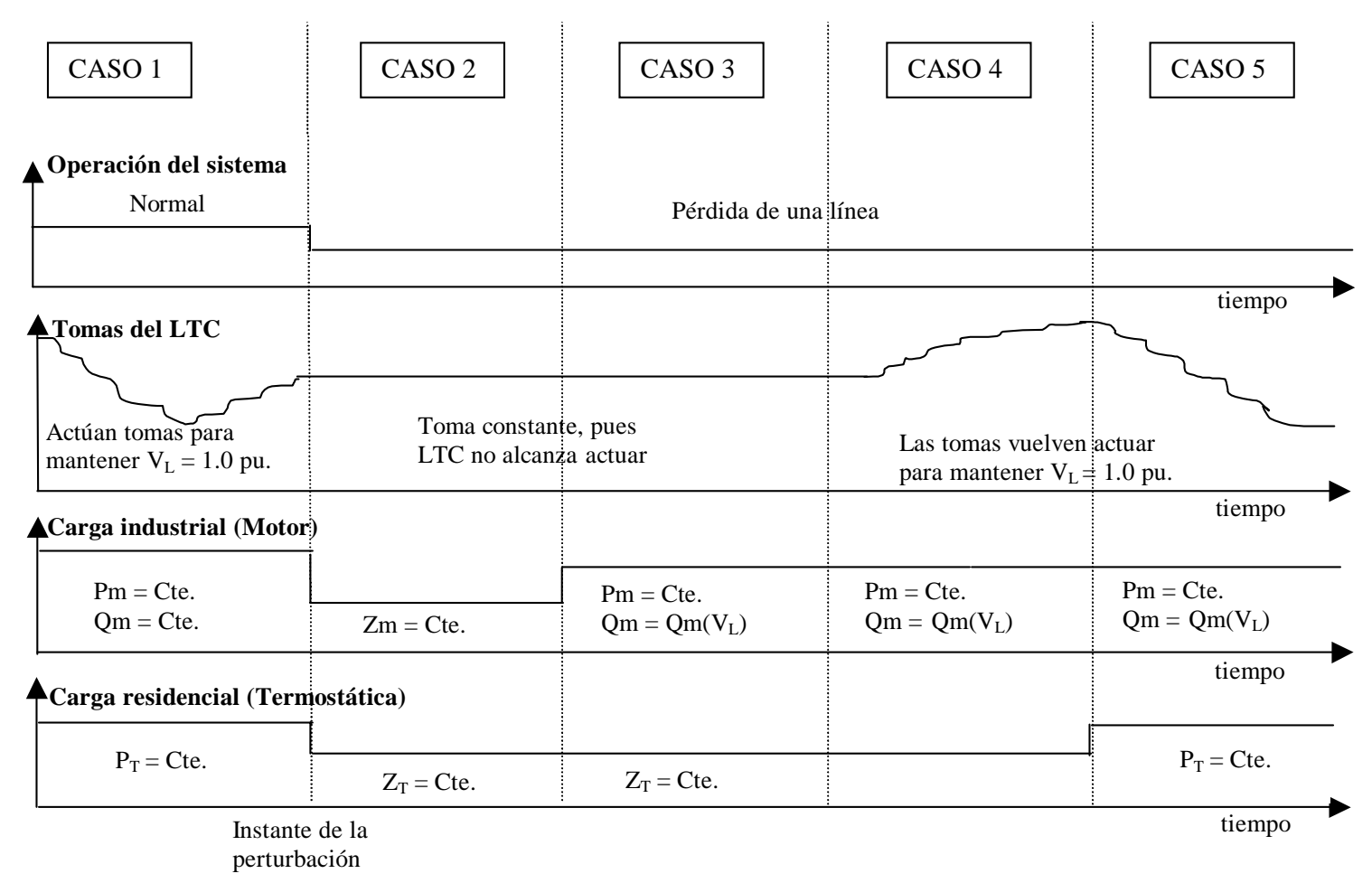

Fig. 2.- Comportamiento de los diferentes elementos del sistema de estudio durante el periodo de tiempo entre pre-falta y post-falta
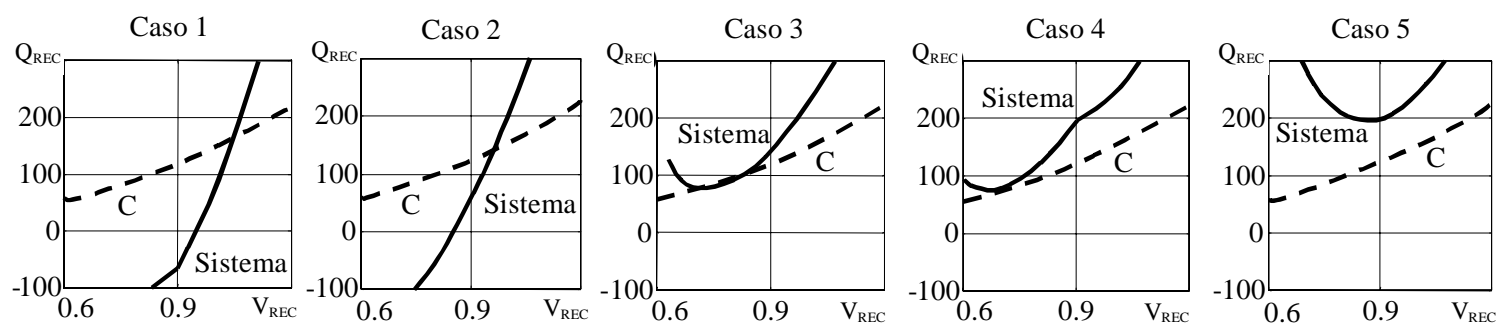

Fig. 3.- Curvas Q-V en el nudo REC para los distintos casos considerados.

C: Característica Q-V del banco de condensadores de 153 MVAr.

Sistema: Característica Q-V del sistema

\section{ESTABILIDAD DE TENSIÓN DESDE UN PUNTO DE VISTA DINÁMICO DE CORTO PLAZO}

A partir de los resultados obtenidos con el análisis cuasi-estático realizados en el punto anterior se ha demostrado que esta metodología es incapaz de realizar una interpretación precisa del problema de estabilidad de tensión, puesto que un estudio más acabado requiere 
el empleo de modelos dinámicos con los cuales sea posible evaluar la evolución de la tensión en el tiempo en una determinada barra del sistema.

\section{Modelos dinámicos de los elementos}

La selección de los modelos de los componentes en un problema de estabilidad de tensión depende fundamentalmente del horizonte de estudio que se quiera estudiar (corto o largo plazo). Como el problema que se quiere analizar es la evolución de la tensión en el nudo REC durante los primeros segundos después de ocurrida la perturbación (corto plazo), es posible considerar que el LTC aun no ha sido capaz de actuar para mantener la tensión constante en la barra, por lo cual es suficiente modelar el transformador en régimen permanente considerando que la toma permanece constante en el valor de pre-falta. En cuanto a la carga residencial se considera que es mayoritariamente de naturaleza resistiva, por lo cual se ha modelado a impedancia constante. El resto de los elementos del sistema sí requieren de un modelo más preciso, los cuales se presentan a continuación.

\section{Carga industrial}

Para el estudio del problema de colapso de tensión en el corto plazo el efecto de las cargas industriales es de especial interés; en este trabajo se ha considerado que la carga industrial se puede representar por un motor de inducción equivalente a través de un modelo de tercer orden (se ha despreciado el comportamiento transitorio del estator) definido por las ecuaciones en p.u (1), (3).

$$
\frac{1}{\mathrm{w}_{\text {base }}} \frac{\mathrm{dv}_{\mathrm{d}}^{\prime}}{\mathrm{dt}}=-\frac{1}{\mathrm{~T}_{0}^{\prime}}\left(\mathrm{v}_{\mathrm{d}}^{\prime}+\left(X-X^{\prime}\right) \mathrm{i}_{\mathrm{q}}^{\mathrm{s}}\right)+\frac{1}{\mathrm{w}_{\text {base }}} \frac{\mathrm{d} \theta_{\mathrm{r}}}{\mathrm{dt}} \mathrm{v}_{\mathrm{q}}^{\prime}
$$

$\frac{1}{\mathrm{w}_{\text {base }}} \frac{\mathrm{dv}_{\mathrm{q}}^{\prime}}{\mathrm{dt}}=-\frac{1}{\mathrm{~T}_{0}^{\prime}}\left(\mathrm{v}_{\mathrm{q}}^{\prime}-\left(X-X^{\prime}\right) \mathrm{i}_{\mathrm{d}}^{\mathrm{s}}\right)-\frac{1}{\mathrm{w}_{\text {base }}} \frac{\mathrm{d} \theta_{\mathrm{r}}}{\mathrm{dt}} \mathrm{v}_{\mathrm{d}}^{\prime}$

$$
2 \mathrm{H} \frac{\mathrm{dw}}{\mathrm{dt}}=\mathrm{T}_{\mathrm{e}}-\mathrm{T}_{\mathrm{o}}\left(\mathrm{Aw}_{\mathrm{m}}^{2}+\mathrm{Bw} \mathrm{w}_{\mathrm{m}}+\mathrm{C}\right)-\mathrm{T}_{\text {roce }}
$$

El modelo del compensador síncrono se ha obtenido a partir del análisis de un motor síncrono que trabaja sin carga (potencia activa nula). Su dinámica se ha modelado con las ecuaciones en p.u. (4) y (5) para el estator en el que se ha despreciado su dinámica y las ecuaciones en p.u. (6), (7) y (8) para el rotor.

$$
\begin{gathered}
{\left[\begin{array}{c}
\mathrm{i}_{\mathrm{d}}^{\mathrm{s}} \\
\mathrm{i}_{\mathrm{q}}^{\mathrm{s}}
\end{array}\right]=\left[\begin{array}{cc}
-\mathrm{R}_{\mathrm{s}} & \mathrm{X}_{\mathrm{q}} \\
-\mathrm{X}_{\mathrm{d}}^{\prime} & -\mathrm{R}_{\mathrm{s}}
\end{array}\right]^{-1} \cdot\left(\left[\begin{array}{c}
\mathrm{v}_{\mathrm{d}}^{\mathrm{s}} \\
\mathrm{v}_{\mathrm{q}}^{\mathrm{s}}
\end{array}\right]-\left[\begin{array}{c}
0 \\
\mathrm{E}_{\mathrm{q}}^{\prime}
\end{array}\right]\right)} \\
\frac{\mathrm{d} \Delta \mathrm{w}_{\mathrm{m}}=\left(\mathrm{X}_{\mathrm{d}}^{\prime}-\mathrm{X}_{\mathrm{d}}\right) \mathrm{i}_{\mathrm{d}}^{\mathrm{s}}-\mathrm{E}_{\mathrm{q}}^{\prime}}{\mathrm{dt}}=-\frac{1}{2 \mathrm{H}}\left(\frac{\mathrm{v}_{\mathrm{d}}^{\prime} \mathrm{i}_{\mathrm{d}}^{\mathrm{s}}+\mathrm{v}_{\mathrm{q}}^{\prime} \mathrm{i}_{\mathrm{q}}^{\mathrm{s}}}{\mathrm{w}_{\mathrm{s}}}\right) \\
\frac{\mathrm{dE} \mathrm{q}_{\mathrm{q}}^{\prime}}{\mathrm{dt}}=\frac{1}{\mathrm{~T}_{\mathrm{do}}^{\prime}} \cdot\left(\mathrm{E}_{\mathrm{fd}}-\mathrm{E}_{\mathrm{l}}\right) \\
\frac{\mathrm{d} \delta}{\mathrm{dt}}=\Delta \mathrm{w}_{\mathrm{m}} \mathrm{w}_{\text {base }} \frac{\mathrm{d} \delta}{\mathrm{dt}}=\Delta \mathrm{w}_{\mathrm{m}} \mathrm{w}_{\text {base }}
\end{gathered}
$$

Para considerar el efecto de la sobreexcitación, el control de la excitación se modela con el bloque mostrado en la Fig. 4, [1].

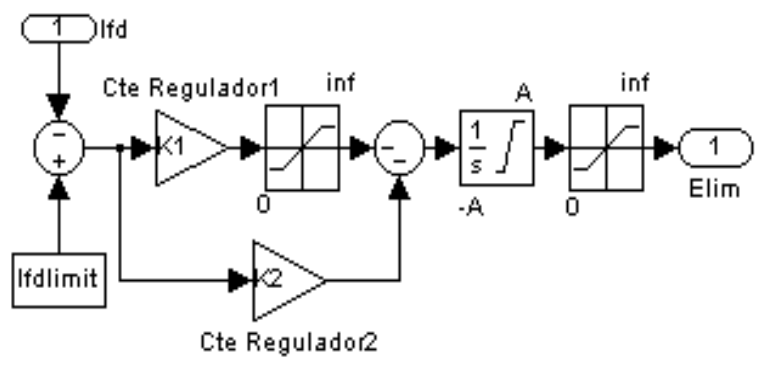

Fig. 4.- Modelo en Simulink para la sobreexcitación del compensador síncrono (CS)

En la Fig. 5 se muestra el diagrama de bloques que relaciona las ecuaciones anteriores mediante un modelo implementado en Simulink

\section{Compensador síncrono (CS)}

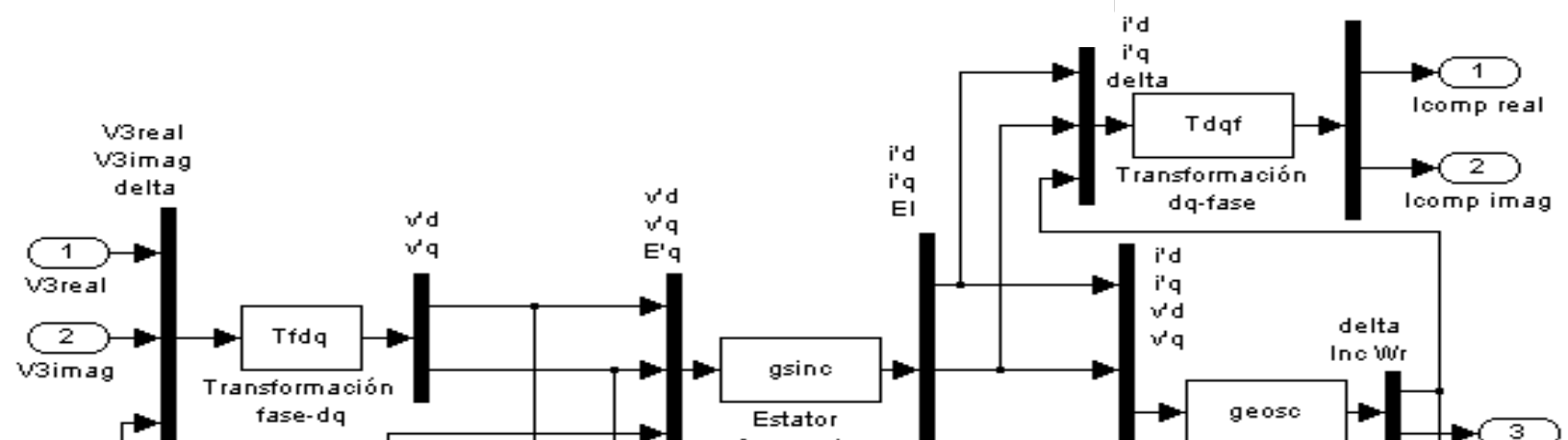




\section{Compensador estático de potencia reactiva (SVC)}

El comportamiento dinámico del SVC se ha realizado con un modelo de primer orden, Fig. 6.

visef.

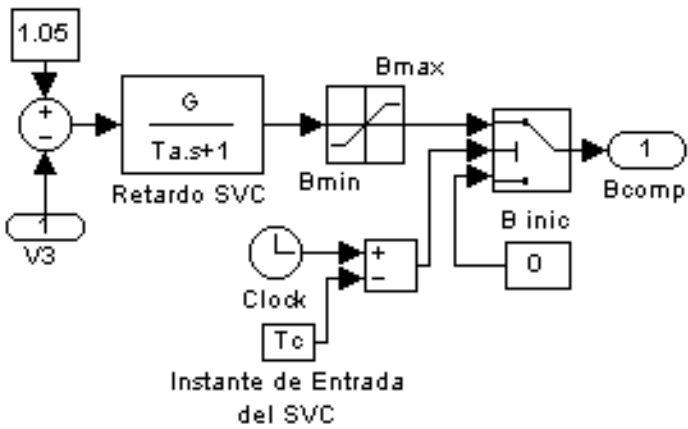

Fig. 6.- Modelo en Simulink para el controlador estático de potencia reactiva $(\mathrm{SVC})$

\section{PROCEDIMIENTO DE SIMULACIÓN DINÁMICA}

Para realizar el estudio en el corto plazo considerando el cortocircuito en el punto medio de una de las líneas (gran perturbación), el sistema de potencia objeto de este estudio ha sido modelado considerando:

- Alimentación desde un nudo de potencia infinita (nudo SOURCE).
- Las líneas y las susceptancias de compensación se consideran de parámetros constantes, ignorando sus transitorios.

- El transformador se considera con su toma fija, pues se supone que las tomas no actúan en los tiempos de simulación considerados en este problema.

- No se considera la dinámica de la carga residencial (análisis en el corto plazo).

- Se consideran los modelos dinámicos del motor de inducción, compensador estático de potencia reactiva (SVC) y compensador síncrono (CS), de acuerdo a los modelos presentados anteriormente.

La Fig. 7 muestra una representación del sistema de estudio, mostrando todos los elementos del mismo.

Es importante destacar que se debe realizar una interconexión entre las partes dinámicas del sistema, aquellas en las que se considera su transitorio, con las partes con comportamiento permanente, es decir en las que no se considera su transitorio.

\section{Interfase Red - Cargas dinámicas}

Para simular la interacción entre la red y las cargas dinámicas, se realiza una interfase que enlace la operación permanente de la red eléctrica con la 
operación transitoria de las distintas cargas dinámicas que existen en la red, para ello, ambos sistemas se relacionan mediante un análisis por nudos considerando el aporte de los elementos dinámicos como fuentes de corriente, tal como se muestra en la Fig. 7, donde las inyecciones de intensidad $\left(\mathrm{I}_{\mathrm{i}}\right)$ se pueden representar como se indica en (9) y los elementos de las submatrices $\left(\mathrm{Y}_{\mathrm{ij}}\right)$ corresponden a los valores de la matriz admitancias de nudos ( $\left.\mathrm{Y}_{\mathrm{BUS}}\right)$ de la red.

$$
\left[\begin{array}{c}
\mathrm{I}_{\mathrm{g}} \\
\mathrm{I}_{2} \\
\mathrm{I}_{3} \\
0
\end{array}\right]=\left[\begin{array}{l|l}
\left(\mathrm{Y}_{\mathrm{gg}}\right) & \left(\mathrm{Y}_{\mathrm{gr}}\right) \\
\hline\left(\mathrm{Y}_{\mathrm{rg}}\right) & \left(\mathrm{Y}_{\mathrm{rr}}\right)
\end{array}\right] \cdot\left[\begin{array}{c}
\varepsilon_{\mathrm{g}} \\
\mathrm{V}^{\prime} \\
\mathrm{V}_{3} \\
\mathrm{~V}_{4}
\end{array}\right]
$$

donde:

$$
Y_{g g}=\left[\begin{array}{cc}
Y_{11} & Y_{12} \\
Y_{21} & Y_{22}
\end{array}\right]
$$

$$
\begin{gathered}
Y_{\mathrm{gr}}=Y_{\mathrm{rg}}=\left[\begin{array}{ll}
\mathrm{Y}_{13} & \mathrm{Y}_{14} \\
\mathrm{Y}_{23} & \mathrm{Y}_{24}
\end{array}\right] \\
Y_{\mathrm{rr}}=\left[\begin{array}{ll}
\mathrm{Y}_{33} & \mathrm{Y}_{34} \\
Y_{43} & Y_{44}
\end{array}\right]
\end{gathered}
$$

de donde:

$$
\left[\begin{array}{l}
\mathrm{I}_{\mathrm{g}} \\
\mathrm{I}_{2}
\end{array}\right]=\left[\mathrm{Y}_{\mathrm{gg}}\right] \cdot\left[\begin{array}{c}
\varepsilon_{\mathrm{g}} \\
\mathrm{V}^{\prime}
\end{array}\right]+\left[\mathrm{Y}_{\mathrm{gr}}\right] \cdot\left[\begin{array}{c}
\mathrm{V}_{3} \\
\mathrm{~V}_{4}
\end{array}\right]
$$

$$
\left[\begin{array}{c}
\mathrm{I}_{3} \\
0
\end{array}\right]=\left[\mathrm{Y}_{\mathrm{rg}}\right] \cdot\left[\begin{array}{c}
\varepsilon_{\mathrm{g}} \\
\mathrm{V}^{\prime}
\end{array}\right]+\left[\mathrm{Y}_{\mathrm{rr}}\right] \cdot\left[\begin{array}{c}
\mathrm{V}_{3} \\
\mathrm{~V}_{4}
\end{array}\right]
$$

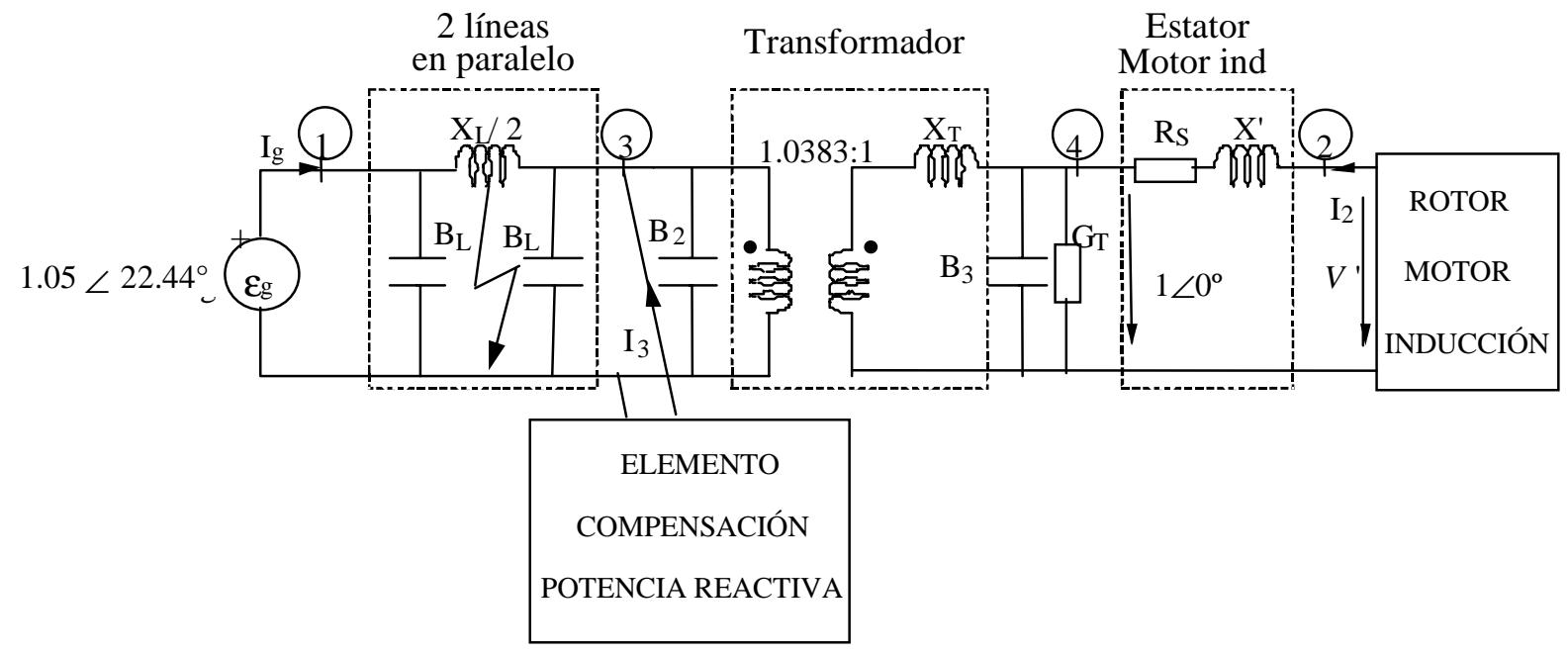

Fig. 7.- Circuito equivalente de la red en estudio

En función del caso particular que se analice, la $\mathrm{Y}_{\mathrm{BUS}} \mathrm{y}$ las corrientes inyectadas tomarán diferentes valores que dependerán de las condiciones de operación del sistema (pre-falta, falta y post-falta) y del tipo de compensación reactiva que se emplee.

El estudio se ha realizado utilizando la herramienta Simulink y Matlab. Para ello se implementó el diagrama de bloques mostrado en la Fig. 8, que se obtuvo considerando el circuito equivalente de la Fig. 7 y las ecuaciones de (1) a (14). En el sitio web www.dinel.upm.es/ hdiaz puede consultarse los 
archivos de simulación así como el detalle teórico de la

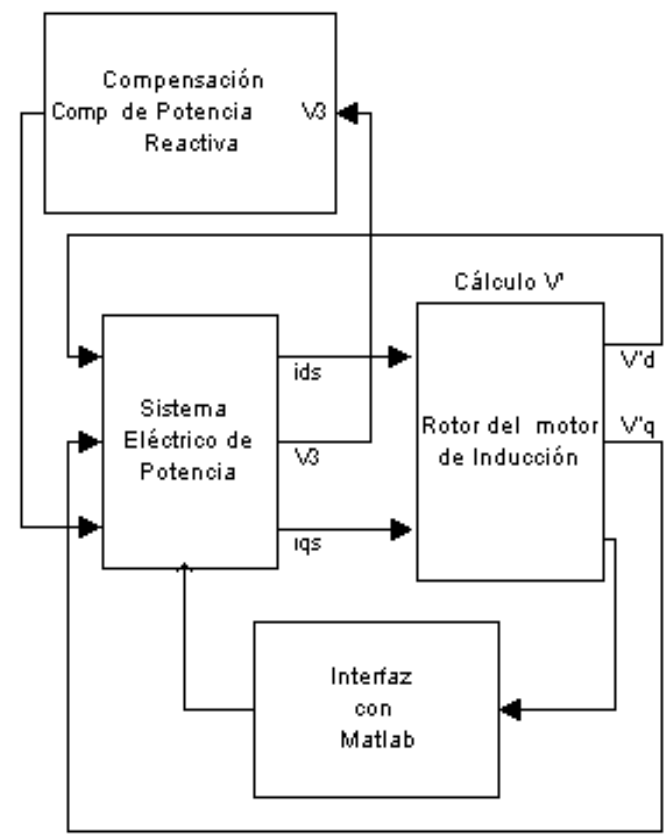

implementación.

Fig. 8.- Diagrama de bloques en Simulink para la simulación dinámica de la red en estudio

\section{RESULTADOS}

Con el fin de comparar los resultados del análisis en el corto plazo obtenidos mediante la simulación cuasiestático (Fig. 3, casos 2, 3) y la simulación dinámica desarrollada, se analiza la respuesta del sistema considerando que la falta tarda 4 ciclos en despejarse y que la dinámica de la carga industrial se representa por un motor de inducción equivalente y la carga residencial por una impedancia constante. Si bien el análisis cuasiestática pareciera no indicar la existencia del fenómeno de colapso de tensión (existe corte entre la curva que representa el sistema y la curva que representa al condensador C), el análisis dinámico, Fig. 9, muestra que sí se produce este fenómeno.

En la Fig. 9c se puede observar que debido a la disminución de tensión el motor es incapaz de suministrar el par eléctrico necesario para satisfacer el par mecánico demandado, causando una disminución sostenida de la velocidad (Fig. 9b) hasta un punto que el motor queda a rotor bloqueado.

Como consecuencia de los resultados anteriores es necesario tomar algunas acciones de control a fin de evitar el problema de colapso de tensión, las dos alternativas clásicas son la inyección de potencia reactiva y el desprendimiento de carga.

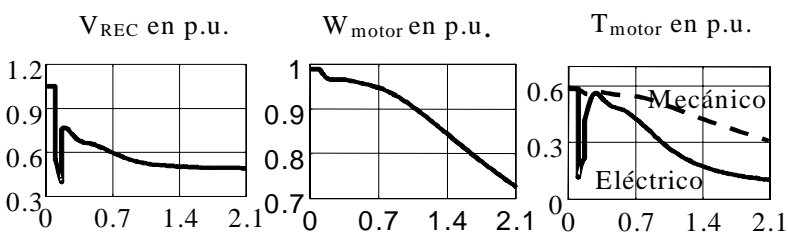

(a)

(b)

(c)

Fig. 9.- Efecto de una falta en una de las líneas sin considerar inyección adicional de potencia reactiva. Escala de tiempos en segundos

\section{Inyección de potencia reactiva}

Para resolver el problema de estabilidad de tensión que se muestra en la Fig. 9 se realiza una inyección de potencia reactiva en el nudo REC con el fin de mantener la tensión de pre-falta, para ello se consideran tres alternativas:

- Banco de condensadores (C) de 125 MVAr que se conecta 0.1 seg. después de despejarse la falta.

- Compensador estático de potencia reactiva (SVC) de +125/-75 MVAr.

- Compensador síncrono (CS) con control de sobreexcitación de 125 MVAr.

En la Fig. 10 se muestra la respuesta del sistema en el nudo REC considerando cada una de estas tres acciones de control, donde puede observarse que en cualquiera de los casos la tensión se recupera después de la perturbación, pero la respuesta dinámica más rápida corresponde al compensador síncrono y la más lenta al banco de condensadores, que además produce una ligera sobretensión. 
Fig. 10.- Evolución de la tensión en el nudo REC (p.u.) con tres alternativas de inyección de potencia reactiva

A continuación se analiza la robustez de estos dispositivos de control frente a situaciones más críticas, como el posible retardo en el instante de conexión a la red y despeje de faltas más lentos.

\section{Influencia del tiempo de conexión del banco de condensadores}

Puesto que el SVC y el compensador síncrono se considera que están permanentemente conectados a la red, sólo se analiza el comportamiento del sistema al conectar el banco de condensadores en diferentes instantes de tiempo. Los resultados obtenidos se muestran en la Fig. 11, donde se observa que esta variable influye fuertemente en la rapidez de respuesta del sistema hasta un instante a partir del cual el banco es incapaz de mantener la estabilidad del sistema.

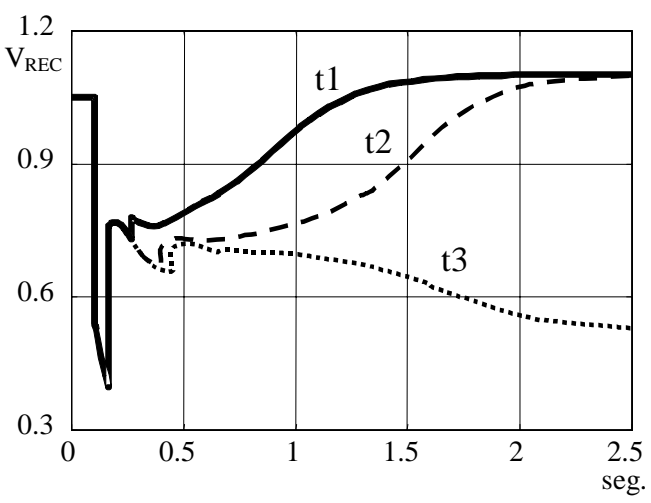

Fig. 11.- Tensión en el nudo REC (p.u.) después del despeje de la falta para distintos instantes de conexión del banco de condensadores $\mathrm{t} 1=0,1$ seg., $\mathrm{t} 2=0,24$ seg., $\mathrm{t} 3=0.29$ seg.

\section{Influencia del tiempo de duración de la falta}

En la Fig. 12 se muestran los tiempos críticos de duración de la falta, que muestran el instante a partir del cual los elementos de control de reactiva son incapaces de mantener la estabilidad de tensión del sistema.

En la Fig. 12a, puede observarse que el banco de condensadores es la solución menos robusta, seguido del SVC (Fig. 12b) y finalmente por el compensador síncrono (Fig. 12d). En este último caso la existencia del límite de sobreexcitación es muy importante, puesto que fija la máxima potencia reactiva que el CS es capaz de entregar, la que tiende a disminuir a medida que aumenta el tiempo, haciendo que este límite sea de interés en análisis de largo plazo.

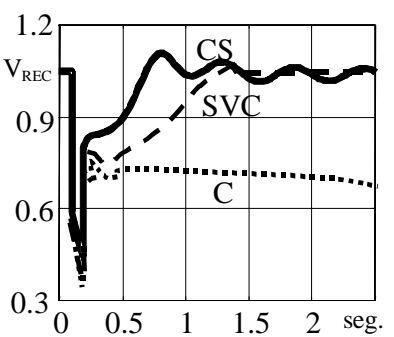

(a)

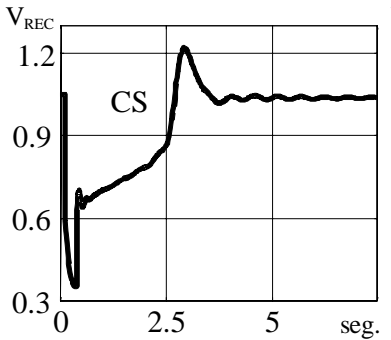

(c)

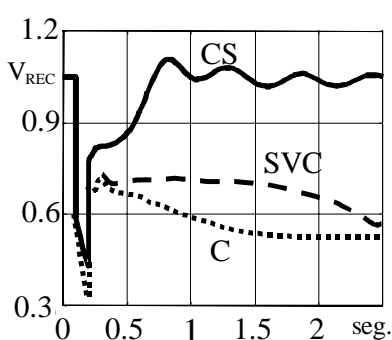

(b)

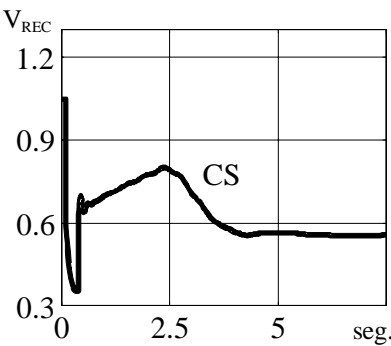

(d)
Fig. 12.- Tensión en el nudo REC (p.u.) para distintos tiempos de duración de la falta

(a) Duración de la falta $=5$ ciclos

(b) Duración de la falta $=5.75$ ciclos

(c) Duración de la falta $=17$ ciclos

(d) Duración de la falta $=18$ ciclos

\section{Desprendimiento de carga}

Como segunda alternativa a la solución del problema de estabilidad de tensión mostrado en la Fig. 9, se plantea el desprendimiento de carga en bloques de igual tamaño. Para ello se considera de forma independiente el efecto del desprendimiento de carga residencial y de carga industrial.

En la Fig. 13 se evalúa la sensibilidad de la tensión en el nudo REC ante el desprendimiento de bloques (Fig. 13a desprendimiento del 10\%, Fig. 13b desprendimiento del $20 \%$ y Fig. 13 c desprendimiento del $30 \%$ ) de carga 
industrial o residencial, considerando que el instante de desprendimiento sucede inmediatamente después del despeje de la falta. Como era de esperar, los resultados muestran que la recuperación de la tensión es altamente sensible a la naturaleza de la carga, comprobándose que el desprendimiento de carga industrial es una mejor alternativa en comparación con el desprendimiento de carga residencial. Por esta razón en la Fig. 14 se presenta una análisis más detallado de esta alternativa, observándose en la Fig. 14a que después de la perturbación, el desprendimiento de carga debe ser lo suficientemente rápido para evitar el colapso de tensión, puesto que el sistema deja ser estable si el instante de desprendimiento supera los 53 ciclos. En la Fig. 13b se observa que el tiempo de duración de la falta también es una variable importante, dado que si éste supera los 15 ciclos el sistema colapsa, independientemente de la rapidez del desprendimiento de la carga después del despeje de la falta.

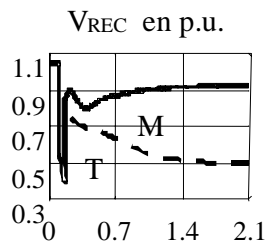

(a)

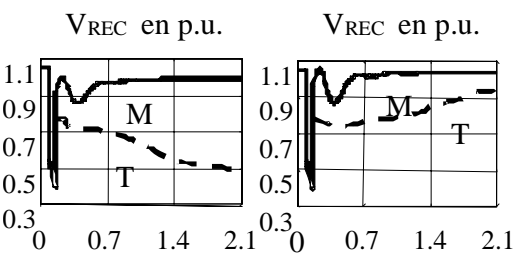

(b)
Fig. 13. - Tensión en el nudo REC considerando distintos niveles de desprendimiento de carga reside ncial (T) o industrial (M) inmediatamente después del desp eje de la falta. Escala de tiempos en segundos

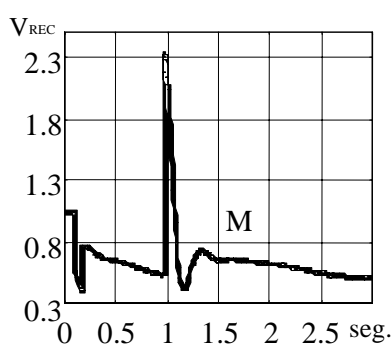

(a)

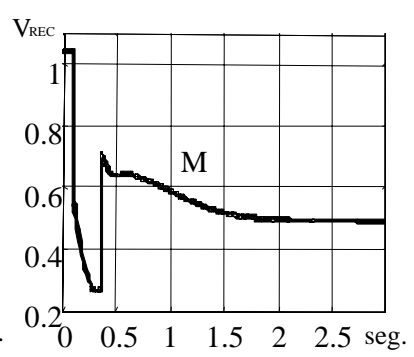

(b)
Fig. 14.- Tensión en el nudo REC (p.u.) con un $10 \%$ de desprendimiento de carga industrial

(a) Instante de desprendimiento $=53$ ciclos

(b) Duración de la falta $=15$ ciclos y desprendimiento inmediatamente después del despeje de la falta

\section{CONCLUSIONES}

En este trabajo, se ha presentado el análisis del comportamiento de diferentes alternativas de control de potencia reactiva y desprendimiento de carga como formas de enfrentar el problema de estabilidad de tensión en el corto plazo. Para ello se ha empleado un sistema obtenido de [1], muy simple pero suficiente para facilitar la comprensión de la problemática y la incidencia del tiempo en la toma de las acciones de control. La simulación se ha realizado empleando rutinas desarrolladas por los autores e implementadas de forma sencilla en Simulink, sin necesidad de tener que contar con un programa específico de análisis de transitorios.

Los resultados corroboran lo obtenido en [1] y muestran que la compensación de potencia reactiva y el desprendimiento de carga tienen un efecto positivo en el problema de estabilidad de tensión; sin embargo, los tiempos asociados a los retardos de la acción de control resultan importantes.

En particular se compara el comportamiento transitorio (corto plazo) entre un compensador síncrono y un SVC, demostrándose que la respuesta dinámica del compensador síncrono es la mejor de las alternativas analizadas, sin embargo también una de las de mayor costo. También se muestra que los bancos de condensadores son una alternativa mucho más económica, pero su efectividad es altamente dependiente del tiempo de conexión. En cuanto al desprendimiento de carga, éste resulta mucho más efectivo mientras más rápido se realice y se obtienen mejores resultados si se aplica a cargas asociadas a motores de inducción.

El detalle teórico y los programas empleados se encuentran disponibles en un tutorial preparado por los autores y que se encuentra disponible en el sitio web www.dinel.upm.es/ hdiaz, lo cual hace que este trabajo pueda servir como apoyo para todos aquellos que deseen iniciarse en el tema del análisis de la estabilidad de tensión de un sistema eléctrico de potencia.

Una situación de interés que queda planteada para futuros trabajos es el estudio de estabilidad de tensión en el largo plazo, para ello debería considerarse un modelo dinámico del LTC y tener en cuenta la dinámica de la carga pasiva.

\section{LISTA DE SÍMBOLOS}

$\begin{array}{ll}\mathrm{w}_{\mathrm{s}} & \text { : Velocidad síncrona del motor. } \\ \mathrm{W}_{\mathrm{m}} & \text { : Velocidad del motor. } \\ \mathrm{W}_{\text {base }} & \text { : Velocidad base. }\end{array}$


$\mathrm{v}_{\mathrm{d}}^{\prime}, \mathrm{v}_{\mathrm{q}}^{\prime} \quad$ : Tensión de eje d y q de entrada al rotor del motor.

$\mathrm{v}_{\mathrm{d}}^{\mathrm{s}}, \mathrm{v}_{\mathrm{q}}^{\mathrm{s}}$ : Tensión de eje d y q del estator del motor.

$\mathrm{i}_{\mathrm{d}}^{\mathrm{s}}, \mathrm{i}_{\mathrm{q}}^{\mathrm{s}} \quad$ : Corrientes de eje d y q del motor.

$\mathrm{T}_{0}^{\prime} \quad$ : Constante de tiempo del rotor.

$\mathrm{X}, \mathrm{X}^{\prime} \quad$ : Parámetros dinámicos del motor.

$\theta_{\mathrm{r}} \quad:$ Ángulo entre el eje "d" y la fase "a" del rotor.bvv

$\mathrm{H} \quad$ : Constante de Inercia del motor.

$\mathrm{T}_{\mathrm{e}} \quad$ : Par eléctrico.

$\mathrm{T}_{0}, \mathrm{~A}, \mathrm{~B}, \mathrm{C}$ : Parámetros del par de la carga

$\mathrm{T}_{\text {roce }} \quad$ : Par de rozamiento.

$\mathrm{R}_{\mathrm{s}} \quad$ : Resistencia del estator del CS.

$\mathrm{X}_{\mathrm{q}} \quad$ : Reactancia de eje $\mathrm{q}$ del CS.

$\mathrm{X}_{\mathrm{d}}, \mathrm{X}_{\mathrm{d}}^{\prime}$ : Reactancia de eje d del CS.

$\mathrm{E}_{\mathrm{q}}^{\prime}, \mathrm{E}_{1}$ : Tensiones internas del CS.

$\delta \quad$ : Posición relativa del rotor del CS.

$\mathrm{T}_{\mathrm{d} 0}^{\prime} \quad$ : Constante de tiempo del CS.

$\mathrm{E}_{\mathrm{fd}} \quad$ : Tensión de excitación del CS.

\section{REFERENCIAS}

[1] Carson W. Taylor; "Power System Voltage Stability"; McGraw-Hill, Inc, 1994.

[2] P. Kundur; "Power System Stability and Control"; McGraw-Hill, Inc, 1994.

[3] T. Van Cutsem; "Voltage Instability: Phenomena, Countermeasures, and Analysis Methods" Proc. IEEE, Vol. 88, $\mathrm{N}^{\circ}$ 2, February 2000.

[4] D. Chattopadhyay et. Al; "Pricing for Voltage Stability" $22^{\circ}$ IEEE International Conference on Power Engineering Society, pp. 235-204, 2001.

[5] A. E. Hammad; "Comparing the Voltage Control Capabilities of Present and Future Var Compensating Techniques in Transmission Systems" IEEE Transaction on Power Delivery, Vol.11, N 1, pp. 475-484, January 1996.

[6] Carson W. Taylor; "Concepts of Undervoltage Load Shedding for Voltage Stability" IEEE Transaction on Power Delivery, Vol. 7, $\mathrm{N}^{\circ}$ 2, pp. 480-488, January 1996.

[7] L. Vargas, C. Cañizares; "Time Dependence of Controls to Avoid Voltage Collapse" IEEE Transaction on Power Systems, Vol. 15, $\mathrm{N}^{\circ} 4$, pp. 1367-1375, July 2000.

[8] B. Chowdhury; C.W. Taylor., "Voltage Stability Analysis: V-Q Power Flow Simulation Versus Dynamic Simulation” IEEE Transaction on Power
Systems, Vol. 15, No 4, pp. 1354-1359, November 2000.

[9] N. Rajakovic, D. Tasic; "Flexible Approach to Dynamic Simulation of Voltage Collapse Phenomenon" ETEP, Vol. 7, N 3, May/June 1997.

[10] M. A. Merkle, A.M. Miri; "Modelling of Industrial Loads for Voltage Stability Studies in Power Systems", Canadian Conference on Electrical and Computer Engineering, pp. 881-886, May 13-16 2001.

[11] V. Ajjarapu, B. Lee; "Bibliography on Voltage Stability" IEEE Transaction on Power Systems, Vol. 13, N 1, pp. 115-125, February 1998. 